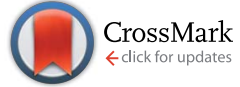

Cite this: Chem. Sci., 2015, 6, 4179

Received 9th February 2015 Accepted 25th April 2015

DOI: $10.1039 / \mathrm{c} 5 \mathrm{sc} 00494 \mathrm{~b}$

www.rsc.org/chemicalscience

\title{
Organocatalytic asymmetric chlorinative dearomatization of naphthols $\uparrow$
}

\author{
Qin Yin, Shou-Guo Wang, Xiao-Wei Liang, De-Wei Gao, Jun Zheng and Shu-Li You*
}

An organocatalytic asymmetric chlorinative dearomatization of naphthols was realized for the first time, providing chiral naphthalenones with a $\mathrm{Cl}$-containing all-substituted stereocenter in excellent yields and enantioselectivity (up to $97 \%$ yield and $96 \%$ ee). The reaction features mild reaction conditions, good tolerance of diverse functional groups and simple reaction operation.

Phenol and its derivatives are readily accessible chemical feedstocks and are widely utilized in chemical synthesis. ${ }^{1}$ Among the versatile transformations, the catalytic asymmetric dearomatization (CADA) reaction ${ }^{2}$ of phenol derivatives offers a facile and straightforward route to access chiral cyclic enones with one quaternary carbon stereogenic center. Therefore, development of the CADA reaction of phenol derivatives has received increasing attention recently. ${ }^{3}$ Strategies for the direct catalytic asymmetric dearomatization of phenols, including hypervalent iodine ${ }^{4}$ or transition metal-catalyzed ${ }^{5}$ oxidation, $^{2}$ transition metal-catalyzed allylic alkylation ${ }^{6}$ or arylation, $^{7}$ and chiral phosphoric acid catalyzed amination, ${ }^{8}$ have been elegantly unveiled. ${ }^{9}$ Very recently, Toste and co-workers reported a highly enantioselective dearomative fluorination of phenols by chiral anion phase-transfer catalysis. ${ }^{10}$ Inspired by these pioneering works, we envisaged that the asymmetric chlorinative dearomatization of phenols via homogeneous catalysis might be possible, providing interesting products with a C-Cl bond-containing chiral center. ${ }^{11}$ However, compared with electrophilic fluorination reagents such as Selectfluor, electrophilic chlorination reagents such as $N$-chlorosuccinimide (NCS) and DCDMH (1,3-dichloro-5,5-dimethylhydantoin) have much higher electrophilic reactivity, which may cause a significant amount of background reaction or undesired reactions such as electrophilic aromatic substitution at the ortho or para-position (the problem of regioselectivity). In addition, the construction of a Cl-containing all-substituted stereocenter with high enantioselectivity via dearomatization of phenols remains underexplored. To test our hypothesis, commercially available cinchonine derivatives such as $(\mathrm{DHQD})_{2}$ PHAL were chosen as chiral catalysts since they are

State Key Laboratory of Organometallic Chemistry, Shanghai Institute of Organic Chemistry, Chinese Academy of Sciences, 345 Lingling Lu, Shanghai 200032, China. E-mail: slyou@sioc.ac.cn; Fax: +86-21-5492-5087

$\dagger$ Electronic supplementary information (ESI) available: Experimental procedures and analysis data for the new compounds. CCDC $1048128((R)-2 t)$ and 1048302 $((R)-2 d)$. For ESI and crystallographic data in CIF or other electronic format see DOI: $10.1039 / \mathrm{c} 5 \mathrm{sc} 00494 \mathrm{~b}$ privileged catalysts for the asymmetric halofunctionalization of alkenes. $^{12,13}$ After extensive preliminary investigation of substituted phenols, we found that naphthols are suitable substrates for the chlorinative dearomatization process. ${ }^{14}$ Herein, we report such a highly enantioselective dearomative chlorination of naphthols under catalysis by (DHQD) ${ }_{2}$ PHAL, providing an efficient synthesis of chiral naphthalenones with an $\alpha$-Cl-containing all-substituted stereocenter (Scheme 1). ${ }^{15}$

We commenced our studies by testing the reactions between commercially available substrate 1a and 1,3-dichloro-5,5-dimethylhydantoin (DCDMH) in the presence of $10 \mathrm{~mol} \%$ (DHQD) ${ }_{2}$ PHAL ${ }^{16}$ Firstly, various solvents were surveyed at room temperature. The dearomative product $\mathbf{2 a}$ could be obtained in $94 \%$ yield in toluene with encouraging enantioselectivity observed ( $52 \%$ ee, entry 1). Further screening of chlorine-containing solvents revealed that $\mathrm{CCl}_{4}$ could give comparable results, affording $2 \mathrm{a}$ in $54 \%$ ee (entries $2-5$ ). To our surprise, the screening of other solvents revealed that $\mathrm{CS}_{2}$ gave the best results and 2 a could be produced in $90 \%$ yield with $62 \%$ ee with a prolonged reaction time (entries 6-8). However, the enantioselectivity of 2a was only slightly elevated from $62 \%$ ee to $64 \%$ ee when the reaction was carried out at $-30{ }^{\circ} \mathrm{C}$ in $\mathrm{CS}_{2}$ (entry 9). Gratifyingly, a significant increase in enantioselectivity could be achieved when the reaction was performed in toluene at a

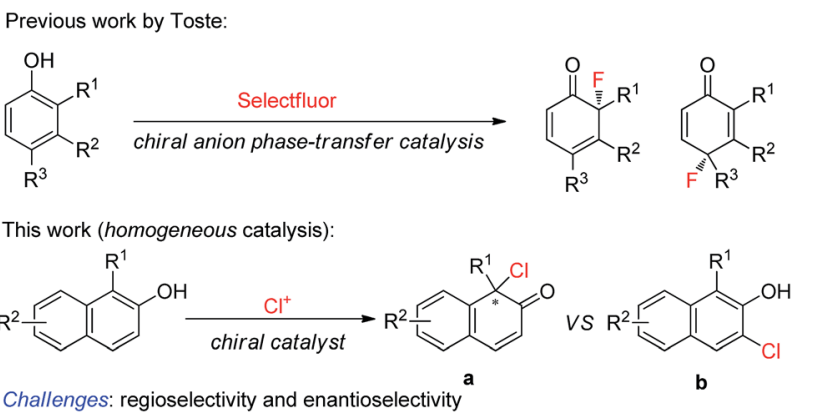

Scheme 1 Asymmetric chlorination of naphthol derivatives via homogeneous catalysis. 
decreased temperature. 2a was obtained in 95\% yield with $92 \%$ ee by carrying out the reaction at $-78{ }^{\circ} \mathrm{C}$ (entry 11). Notably, by changing the catalyst from (DHQD) ${ }_{2} \mathrm{PHAL}$ to (DHQ) ${ }_{2} \mathrm{PHAL}, 2 \mathrm{a}$ with $90 \%$ ee, with the opposite configuration, could be obtained in an almost quantitative yield (entry 12). A decrease in the catalyst loading from $10 \mathrm{~mol} \%$ to $2 \mathrm{~mol} \%$ led to a prolonged reaction time, however, the yield and enantioselectivity of $\mathbf{2 a}$ remained at an excellent level (98\% yield, 90\% ee, entry 13) (Table 1).

Under the optimized reaction conditions, 2-naphthols with different substituents were synthesized to test the generality of this asymmetric chlorination process (Scheme 2). Firstly, the substituent effect of the ester group (Me, Et, allyl) was evaluated and, in all cases, excellent yields were achieved. With the increase in steric hindrance from a methyl, to an ethyl, to an allyl group, the enantioselectivity of the corresponding products 2a-2c showed a decreasing trend. However, the levels were still excellent (2a-2c, 91-95\% yields, 86-92\% ee). The substituent effect on the core of 2-hydroxy-1-naphthoate was next investigated. Electron-withdrawing groups such as 6-Br and 6-CN, or aryl groups such as $\mathrm{Ph}, 3,5-(\mathrm{Me})_{2} \mathrm{C}_{6} \mathrm{H}_{3}$ and $4-\mathrm{F}_{-} \mathrm{C}_{6} \mathrm{H}_{4}$ were well tolerated. The corresponding products were all obtained in excellent yields and enantioselectivity (2d-2h, $80-88 \%$ yields, 93-96\% ee). Electron-donating groups such as 6-Me and 6-phenethyl were also well tolerated and the corresponding

Table 1 Evaluation of reaction conditions

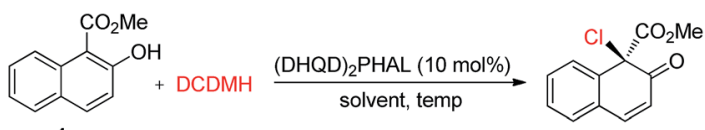

1a

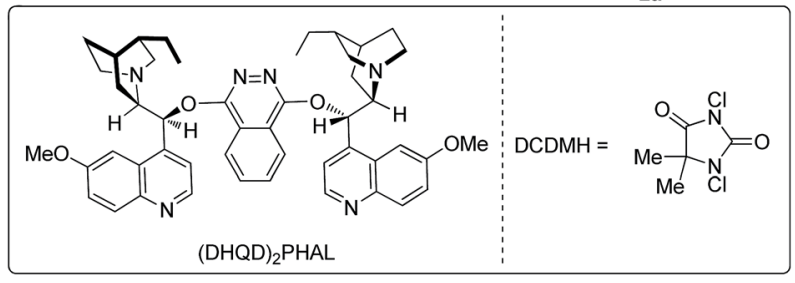

\begin{tabular}{llllll}
\hline Entry $^{a}$ & Solvent & Temp $\left({ }^{\circ} \mathrm{C}\right)$ & Time (h) & Yield $^{b}(\%)$ & Ee $\left.^{c} \%\right)$ \\
\hline 1 & Toluene & $\mathrm{Rt}$ & 1 & 94 & 52 \\
2 & DCM & $\mathrm{Rt}$ & 1 & 92 & 36 \\
3 & $\mathrm{DCE}$ & $\mathrm{Rt}$ & 1 & 93 & 25 \\
4 & $\mathrm{CHCl}_{3}$ & $\mathrm{Rt}$ & 1 & 92 & 34 \\
5 & $\mathrm{CCl}_{4}$ & $\mathrm{Rt}$ & 1 & 92 & 54 \\
6 & $\mathrm{Hexane}$ & $\mathrm{Rt}$ & 1 & 89 & 32 \\
7 & $\mathrm{THF}$ & $\mathrm{Rt}$ & 1 & 88 & 12 \\
8 & $\mathrm{CS}_{2}$ & $\mathrm{Rt}$ & 4 & 90 & 62 \\
9 & $\mathrm{CS}_{2}$ & -30 & 24 & 91 & 64 \\
10 & Toluene & -30 & 8 & 94 & 75 \\
11 & Toluene & -78 & 10 & 95 & 92 \\
$12^{d}$ & Toluene & -78 & 10 & 94 & -90 \\
$13^{e}$ & Toluene & -78 & 31 & 98 & 90
\end{tabular}

${ }^{a}$ Reactions were performed with 1a $(0.1 \mathrm{mmol})$, DCDMH $(0.12 \mathrm{mmol})$ and $10 \mathrm{~mol} \%$ of (DHQD) ${ }_{2}$ PHAL at $\mathrm{rt}$ in an open flask. ${ }^{b}$ Isolated yield. ${ }^{c}$ Determined by HPLC analysis. ${ }^{d} 10 \mathrm{~mol} \%$ of (DHQ) ${ }_{2}$ PHAL was utilized. $^{e} 2$ mol\% of (DHQD) ${ }_{2}$ PHAL was utilized.
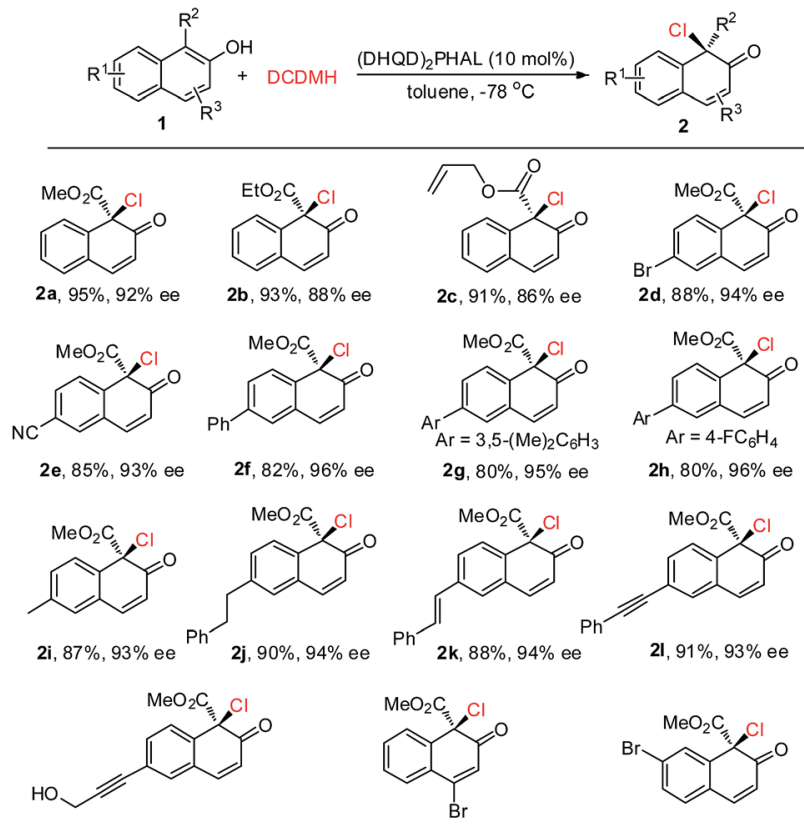

21, $91 \%, 93 \%$ ee

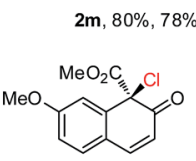

2 p, $95 \%, 90 \%$ ee

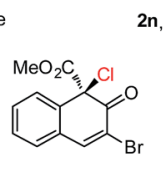

2q. $88 \%, 73 \%$ ee 2n, $90 \%, 94 \%$ ee

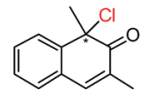

2 r, $91 \%, 82 \%$ ee $94 \%, 86 \%$ ee

a $10 \mathrm{~mol} \%$ of $(\mathrm{DHQ})_{2} \mathrm{PHAL}$ was utilized.
Scheme 2 Evaluation of substrate scope.

products $2 \mathbf{i}$ and $2 \mathbf{j}$ were obtained in $87 \%$ yield, $93 \%$ ee and $90 \%$ yield, $94 \%$ ee, respectively. To our delight, the unsaturated double bond or triple bond in the substrates did not interfere with the reactivity or enantiocontrol of the reaction. For instance, reactions with substrate $\mathbf{1 k}$ with a styryl group and substrate $\mathbf{1 l}$ with a phenylethynyl group could proceed smoothly to give the corresponding products $2 \mathbf{k}$ and $2 \mathbf{l}$ in $88 \%$ yield, $94 \%$ ee and 91\% yield, 93\% ee, respectively. Furthermore, substrate 1m with both a triple bond and a hydroxyl group was also well tolerated, and product $\mathbf{2 m}$ was obtained in $80 \%$ yield with $78 \%$ ee. Various substituents at other positions of 2-hydroxy-1naphthoate were also surveyed. Substrate 1n with 4-Br, substrate 10 with 7-Br and substrate 1p with 7-MeO could all be successfully converted to their corresponding products in excellent yields and enantioselectivity (2n-2p, 85-95\% yields, 90-94\% ee). In addition, product $2 q$ with a $3-\mathrm{Br}$ substituent was obtained in $88 \%$ yield with $73 \%$ ee. Apart from 2-hydroxy-1naphthoates, 2-naphthols with an electron-donating group at the 1-position were also suitable substrates. For instance, substrate 1r with 1,3-dimethyl groups could be smoothly transformed to the corresponding product $2 \mathbf{r}$ (91\% yield, $82 \%$ ee). To be noted, in the presence of (DHQ) ${ }_{2} \mathrm{PHAL}$, substrates $\mathbf{1 r}$ and $1 \mathrm{~s}$ with 1-Me and 3-Ph, respectively, could also work well in this reaction to yield 2 r (94\% yield, $86 \%$ ee) and 2 s (89\% yield, $82 \%$ ee) respectively. The absolute configuration of the product was determined, by X-ray analysis of enantiopure $\mathbf{2 d}$, as $R$ (see the $\mathrm{ESI} \dagger$ for details). 
Besides 2-naphthols, methyl 1-hydroxy-2-naphthoate, 1t, was also well tolerated in this dearomative chlorination reaction. Under slightly optimized conditions (in the presence of 10 mol\% of (DHQD) $)_{2} \mathrm{PYR}$ in $\mathrm{CHCl}_{3} / \mathrm{CCl}_{4}$ at $-70{ }^{\circ} \mathrm{C}$ ), product $2 \mathrm{t}$ was obtained in $94 \%$ yield with $90 \%$ ee (Scheme 3 ), and its structure was confirmed by X-ray analysis. To our knowledge, highly enantioselective intermolecular dearomatization of 1-naphthol derivatives has not been reported yet. ${ }^{4 b}$

We also tested the asymmetric bromination of 1a with 1,3dibromo-5,5-dimethylhydantoin (DBDMH) under the standard reaction conditions (eqn (1), Scheme 4). The desired brominative product $2 \mathbf{u}$ was obtained in $96 \%$ yield with $9 \%$ ee. The almost racemic result was possibly due to the very strong background reaction. To our surprise, a further attempt using 2hydroxy-1-naphthoic acid (1v) as the substrate in the presence of (DHQD) ${ }_{2}$ PHAL provided the achiral decarboxylative compound $2 \mathbf{v}$ in quantitative yield (eqn (2), Scheme 4).

To evaluate the practicality of this dearomative strategy, gram-scale reactions of $\mathbf{1 a}$ and $\mathbf{1 t}$ were performed. As displayed in Scheme 5, the corresponding products $2 \mathbf{a}$ and $2 \mathbf{t}$ could be obtained in excellent yields without a notable reduction in the enantioselectivity ( $91 \%$ ee and $87 \%$ ee, respectively).

To further show the synthetic utility of this newly developed protocol, several transformations of the products were carried out (Scheme 6). With different workup procedures, 2a could be converted to the chiral allylic alcohol $\mathbf{3 a}$ or epoxide $\mathbf{3} \mathbf{b}$ in moderate yields with excellent diastereoselectivity $(>20: 1)$ via the reduction of carbonyl by Dibal-H (eqn (1) and (2), Scheme 6). When 2a was subjected to oxidative bromination conditions, 2q could be achieved in $74 \%$ yield with $89 \%$ ee, serving as a complementary route to access $\mathbf{2 q}$ with high enantioselectivity (eqn (3), Scheme 6). In addition, 2t could be converted to highly functionalized compounds through stereoselective halogenation of the double bond. For instance, the dibromination product $3 \mathbf{c}$ could be obtained as a single diastereoisomer under bromination conditions, without reduction in the enantioselectivity (85\% yield, 88\% ee, eqn (4), Scheme 6). Furthermore, multi-functionalized chlorohydrin 3d was obtained under electrophilic chlorination reaction conditions in $70 \%$ yield with good stereochemical integrity and high diastereoselectivity $(\mathrm{dr}=11: 1,86 \%$ ee for the major diastereoisomer, eqn (5), Scheme 6). ${ }^{17}$

As for the working model of this reaction, inspired by the pioneering studies by Nicolaou, ${ }^{13 c}$ Hennecke ${ }^{13 k}$ and Tang, ${ }^{13 l}$ we speculate that the phthalazine nitrogen in the catalyst interacts with the hydroxyl group of naphthol via a hydrogen bond to increase the nucleophilic property of the 1-position (Fig. 1). In addition, the intramolecular hydrogen bond of 1a itself also makes a contribution to a relatively rigid chiral environment.

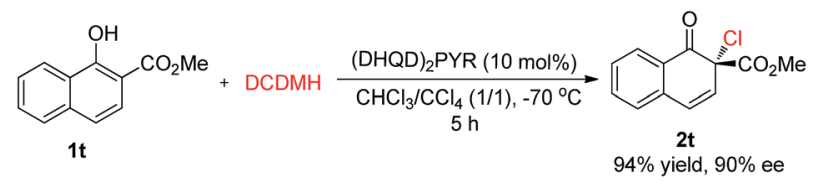

Scheme 3 Asymmetric chlorinative dearomatization reaction of a 1naphthol derivative.

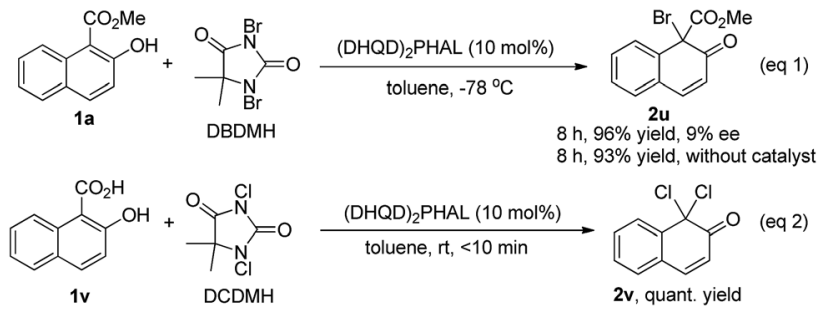

Scheme 4 Bromination of 1a with DBDMH (eqn 1) and reaction of 2hydroxy-1-naphthoic acid (1v) with DCDMH (eqn 2).

On the other hand, the tertiary amine nitrogen in quinuclidine acts as a Lewis base to activate the chloronium species to provide a bifunctional catalytic model, which is in line with Borhan's research. ${ }^{13 a}$

To investigate this proposal, several control experiments were carried out, as shown in Scheme 7. Firstly, when substrate 1u, of which the hydroxyl was protected by a methyl group, was subjected to the chlorination conditions, no reaction occurred (eqn (1), Scheme 7). When the protecting group was changed to TMS, the reaction of $\mathbf{1 v}$ proceeded very slowly to give the desired product $2 \mathrm{a}$ in only $45 \%$ yield with $20 \%$ ee (eqn (2), Scheme 7 ). When a homogeneous toluene solution of the potassium salt of 1a, prepared in situ by treating 1a with 1.05 equiv of KOMe and 18-crown-6, was subjected to the standard conditions, product 2a was obtained in 99\% yield, however in an almost racemic form (eqn (3), Scheme 7). The control experiment (eqn (4), Scheme 7) revealed that the addition of methanol and 18-crown6 did not have any effect on the yield or enantioselectivity of $2 a$. All these experiments suggested that the hydroxyl group in the substrate is relevant not only to the reactivity but also to the enantiocontrol, possibly playing a role as a hydrogen bond donor. Furthermore, the addition of benzoic acid dramatically decreased the reaction rate as well as the enantioselectivity of $2 \mathbf{a}$ from $92 \%$ ee to $64 \%$ ee (eqn (5), Scheme 7). The possible protonation of the quinuclidine nitrogen atom by the acid decreased the catalytic efficiency of the catalyst. Despite the fact that some promising experimental evidence was obtained, the working model is postulated and needs further studies.

In summary, we have realized for the first time the organocatalytic asymmetric chlorinative dearomatization of naphthols, providing chiral naphthalenones with a Cl-containing allsubstituted stereocenter in excellent yields and enantioselectivity. The reaction features mild reaction conditions, good

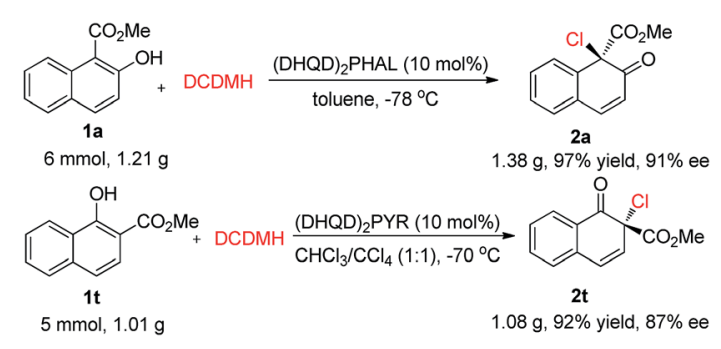

Scheme 5 Gram-scale reactions. 

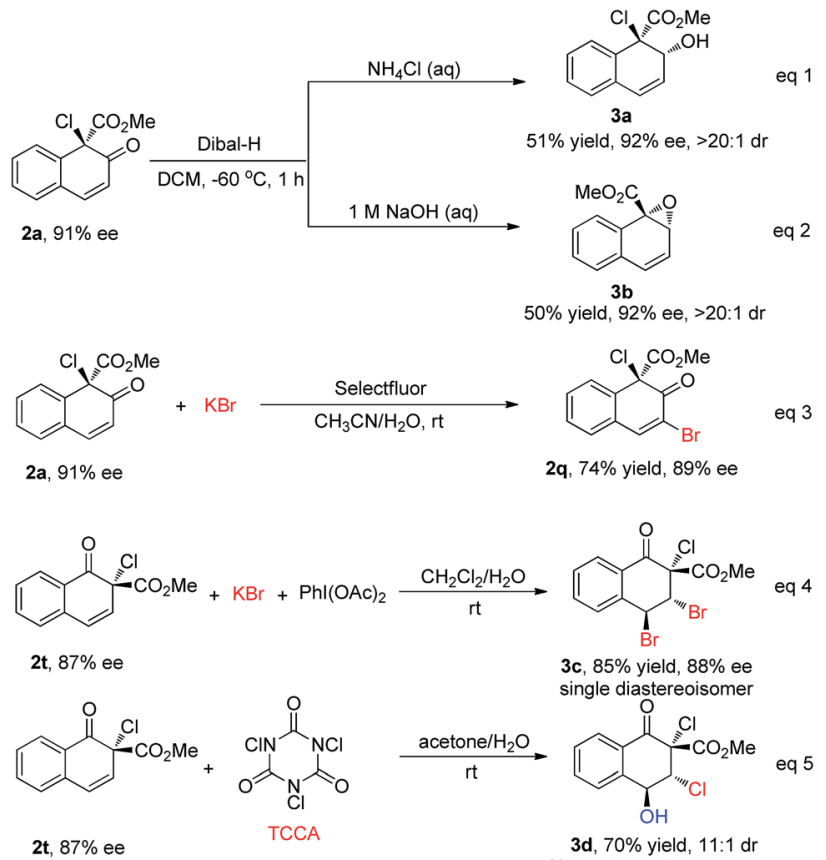

$86 \%$ ee (major diastereoisomer)

Scheme 6 Transformations of products.

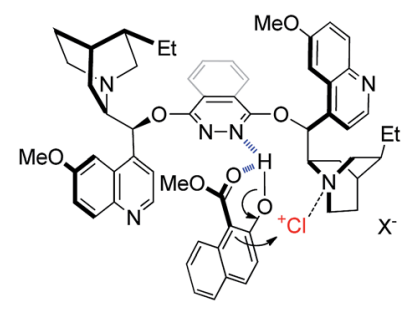

Fig. 1 Proposed working model.

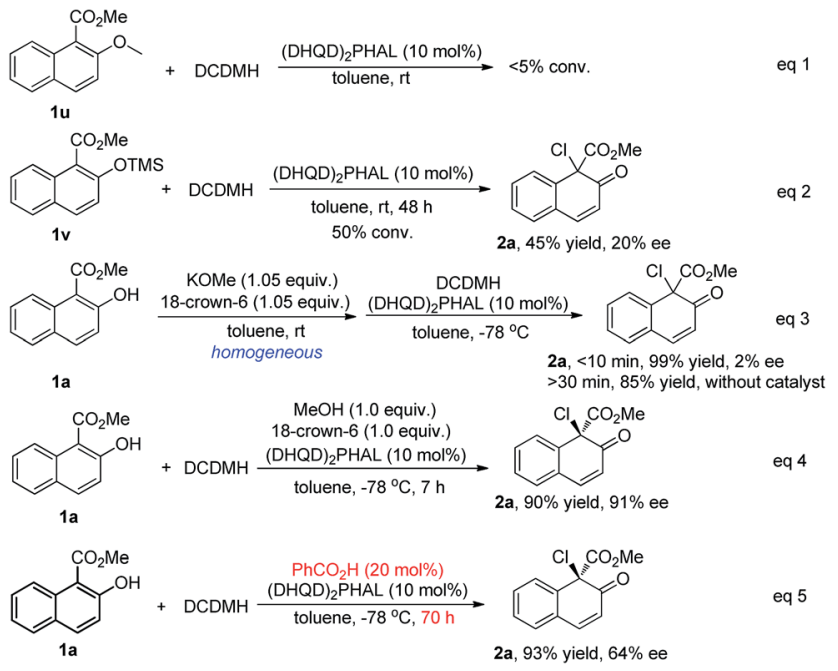

Scheme 7 Control experiments. tolerance of diverse functional groups and simple reaction operation. Notably, highly enantioselective intermolecular dearomative chlorination of 1-naphthol derivative was also realized. In addition, the gram-scale reactions and practical transformations of the products reveal the potential synthetic utility of this method.

\section{Acknowledgements}

We thank the National Basic Research Program of China (973 Program 2015CB856600), the National Natural Science Foundation of China (21332009, 21421091, 21361140373), and the Chinese Academy of Sciences for generous financial support.

\section{Notes and references}

1 M. Weber, M. Weber and M. Kleine-Boymann, "Phenol" in Ullmann's Encyclopedia of Industrial Chemistry, Wiley-VCH, Weinheim, 2004, pp. 503-519.

2 For reviews, see: (a) S. P. Roche and J. A. Porco Jr, Angew. Chem., Int. Ed., 2011, 50, 4068; (b) L. Pouységu, D. Deffieux and S. Quideau, Tetrahedron, 2011, 66, 2235; (c) C.-X. Zhuo, W. Zhang and S.-L. You, Angew. Chem., Int. Ed., 2012, 51, 12662.

3 For selected examples of stepwise asymmetric dearomatization of phenols, see: (a) R. Imbos, A. J. Minnaard and B. L. Feringa, J. Am. Chem. Soc., 2002, 124, 184; (b) Y. Hayashi, H. Gotoh, T. Tamura, H. Yamaguchi, R. Masui and M. Shoji, J. Am. Chem. Soc., 2005, 127, 16028; (c) Q. Liu and T. Rovis, J. Am. Chem. Soc., 2006, 128, 2552; (d) N. T. Vo, R. D. M. Pace, F. O'Hara and M. J. Gaunt, J. Am. Chem. Soc., 2008, 130, 404; (e) Q. Gu, Z.-Q. Rong, C. Zheng and S.-L. You, J. Am. Chem. Soc., 2010, 132, 4056; $(f)$ Q. Gu and S.-L. You, Chem. Sci., 2011, 2, 1519; (g) Q. Gu and S.-L. You, Org. Lett., 2011, 13, 5192; $(h)$ R. Leon, A. Jawalekar, T. Redert and M. J. Gaunt, Chem. Sci., 2011, 2, 1487; (i) M.-Q. Jia and S.-L. You, Chem. Commun., 2012, 48, 6363; (j) M.-Q. Jia, C. Liu and S.-L. You, J. Org. Chem., 2012, 77, 10996; (k) D. M. Rubush, M. A. Morges, B. J. Rose, D. H. Thamm and T. Rovis, J. Am. Chem. Soc., 2012, 134, 13554; (l) S. Takizawa, T. M. Nguyen, A. Grossmann, D. Enders and H. Sasai, Angew. Chem., Int. Ed., 2012, 51, 5423; $(m)$ W. Wu, X. Li, H. Huang, X. Yuan, J. Lu, K. Zhu and J. Ye, Angew. Chem., Int. Ed., 2013, 52, 1743; (n) M. T. Corbett and J. S. Johnson, Chem. Sci., 2013, 4, 2828; (o) Z.-T. He, B. Tian, Y. Fukui, X. Tong, P. Tian and G.-Q. Lin, Angew. Chem., Int. Ed., 2013, 52, 5314.

4 For selected examples, see: (a) T. Dohi, A. Maruyama, N. Takenaga, K. Senami, Y. Minamitsuji, H. Fujioka, S. B. Caemmerer and Y. Kita, Angew. Chem., Int. Ed., 2008, 47, 3787; (b) S. Quideau, G. Lyvinec, M. Marguerit, K. Bathany, A. Ozanne-Beaudenon, T. Buffeteau, D. Cavagnat and A. Chénedé, Angew. Chem., Int. Ed., 2009, 48, 4605; (c) M. Uyanik, T. Yasui and K. Ishihara, Angew. Chem., Int. Ed., 2010, 49, 2175; (d) M. Uyanik, T. Yasui and K. Ishihara, Tetrahedron, 2010, 66, 5841; (e) T. Dohi, N. Takenaga, T. Nakae, Y. Toyoda, M. Yamasaki, M. Shiro, 
H. Fujioka, A. Maruyama and Y. Kita, J. Am. Chem. Soc., 2013, 135, 4558.

5 (a) S. Dong, J. Zhu and J. A. Porco Jr, J. Am. Chem. Soc., 2008, 130, 2738; (b) A. Rudolph, P. H. Bos, A. Meetsma, A. J. Minnaard and B. L. Feringa, Angew. Chem., Int. Ed., 2011, 50, 5834; (c) T. Oguma and T. Katsuki, J. Am. Chem. Soc., 2012, 134, 20017; (d) T. Oguma and T. Katsuki, Chem. Commun., 2014, 50, 5053.

6 (a) T. Nemoto, Y. Ishige, M. Yoshida, Y. Kohno, M. Kanematsu and Y. Hamada, Org. Lett., 2010, 12, 5020; (b) Q.-F. Wu, W.-B. Liu, C.-X. Zhuo, Z.-Q. Rong, K.-Y. Ye and S.-L. You, Angew. Chem., Int. Ed., 2011, 50, 4455; (c) C.-X. Zhuo and S.-L. You, Angew. Chem., Int. Ed., 2013, 52, 10056.

7 (a) S. Rousseaux, J. García-Fortanet, M. A. Del Aguila Sanchez and S. L. Buchwald, J. Am. Chem. Soc., 2011, 133, 9282; (b) R.-Q. Xu, Q. Gu, W.-T. Wu, Z.-A. Zhao and S.-L. You, J. Am. Chem. Soc., 2014, 136, 15469.

8 S.-G. Wang, Q. Yin, C.-X. Zhuo and S.-L. You, Angew. Chem., Int. Ed., 2015, 54, 647.

9 During the preparation of this manuscript, two examples of Lewis acid catalyzed asymmetric dearomatization of naphthols were reported, see: (a) J. Nan, J. Liu, H. Zheng, Z. Zuo, L. Hou, H. Hu, Y. Wang and X. Luan, Angew. Chem., Int. Ed., 2015, 54, 2356; (b) D. Yang, L. Wang, F. Han, D. Li, D. Zhao and R. Wang, Angew. Chem., Int. Ed., 2015, 54, 2185.

10 R. J. Phipps and F. D. Toste, J. Am. Chem. Soc., 2013, 135, 1268.

11 L. Benati, D. Nanni, C. Sangiorgi and P. Spagnolo, J. Org. Chem., 1999, 64, 7836.

12 For selected reviews on asymmetric halogenation of olefins, see: (a) G. Chen and S. Ma, Angew. Chem., Int. Ed., 2010, 49, 8306; (b) A. Castellanos and S. P. Fletcher, Chem.-Eur. J., 2011, 17, 5766; (c) S. E. Denmark, W. E. Kuester and M. T. Burk, Angew. Chem., Int. Ed., 2012, 51, 10938; (d) U. Hennecke, Chem.-Asian J., 2012, 7, 456; (e) K. Murai and H. Fujioka, Heterocycles, 2013, 87, 763; (f) C. K. Tan and Y.-Y. Yeung, Chem. Commun., 2013, 49, 7985; $(g)$ Y. A. Cheng, W. Z. Yu and Y.-Y. Yeung, Org. Biomol. Chem., 2014, 12, 2333; (h) S. Zheng, C. M. Schienebeck, W. Zhang, H.-Y. Wang and W. Tang, Asian J. Org. Chem., 2014, 3, 366.
13 For selected examples of asymmetric halogenation of olefins catalyzed by cinchonine derivatives, see: (a) D. C. Whitehead, R. Yousefi, A. Jaganathan and B. Borhan, J. Am. Chem. Soc., 2010, 132, 3298; (b) W. Zhang, S. Zheng, N. Liu, J. B. Werness, I. A. Guzei and W. Tang, J. Am. Chem. Soc., 2010, 132, 3664; (c) K. C. Nicolaou, N. L. Simmons, Y. Ying, P. M. Heretsch and J. S. Chen, J. Am. Chem. Soc., 2011, 133, 8134; (d) O. Lozano, G. Blessley, T. Martinez del Campo, A. L. Thompson, G. T. Giuffredi, M. Bettati, M. Walker, R. Borman and V. Gouverneur, Angew. Chem., Int. Ed., 2011, 50, 8105; (e) A. Jaganathan, A. Garzan, D. C. Whitehead, R. J. Staples and B. Borhan, Angew. Chem., Int. Ed., 2011, 50, 2593; (f) Z.-M. Chen, Q.-W. Zhang, Z.-H. Chen, H. Li, Y.-Q. Tu, F.-M. Zhang and J.-M. Tian, J. Am. Chem. Soc., 2011, 133, 8818; $(g)$ H. Li, F.-M. Zhang, Y.-Q. Tu, Q.-W. Zhang, Z.-M. Chen, Z.-H. Chen and J. Li, Chem. Sci., 2011, 2, 1839; (h) K. Ikeuchi, S. Ido, S. Yoshimura, T. Asakawa, M. Inai, Y. Hamashima and T. Kan, Org. Lett., 2012, 14, 6016; (i) A. Jaganathan, R. J. Staples and B. Borhan, J. Am. Chem. Soc., 2013, 135, 14806; (j) W. Zhang, N. Liu, C. M. Schienebeck, K. Decloux, S. Zheng, J. B. Werness and W. Tang, Chem.-Eur. J., 2012, 18, 7296; (k) M. Wilking, C. Mück-Lichtenfeld, C. G. Daniliuc and U. Hennecke, J. Am. Chem. Soc., 2013, 135, 8133; (l) W. Zhang, N. Liu, C. M. Schienebeck, X. Zhou, I. I. Izhar, I. A. Guzei and W. Tang, Chem. Sci., 2013, 4, 2652; (m) Q. Yin and S.-L. You, Org. Lett., 2013, 15, 4266; (n) Q. Cai, Q. Yin and S.-L. You, Asian J. Org. Chem., 2014, 3, 408; (o) Q. Yin and S.-L. You, Org. Lett., 2014, 16, 1810; ( $p$ ) Q. Yin and S.-L. You, Org. Lett., 2014, 16, 2426; (q) M. Wilking, C. G. Daniliuc and U. Hennecke, Synlett, 2014, 1701.

14 The dearomatized products of phenol derivatives could be observed by NMR study, but unstable to be isolated.

15 For examples of catalytic asymmetric chlorination of carbonyl compounds, see: K. Shibatomi and A. Narayama, Asian J. Org. Chem., 2013, 2, 812, and references therein.

$16 \mathrm{~N}$-Chlorosuccinimide was also tested as an electrophilic chlorination reagent, however, the reaction was slow and took several days for complete conversion.

17 The relative configurations of products $\mathbf{3 c}$ and $\mathbf{3 d}$ were determined by NOESY NMR analysis, see the $\mathrm{ESI}^{\dagger}$ 